\title{
ANDREEV REFLECTION AND THE SEMICLASSICAL BOGOLIUBOV-DE GENNES HAMILTONIAN: RESONANT STATES
}

\author{
A. BENSOUISSI ${ }^{1,2}$, N. M'HADBI ${ }^{2} \& \mathrm{M}^{\prime}$. ROULEUX ${ }^{1}$ \\ ${ }^{1}$ Université du Sud Toulon-Var, and Centre de Physique Théorique \\ CPT, Case 907, 13288 Marseille Cedex 9, France \\ ${ }^{2}$ Université de Tunis El-Manar, Département de Mathématiques, 1091 Tunis, Tunisia
}

\begin{abstract}
We present a semi-classical analysis of the opening of superchannels in gated mesoscopic SNS junctions. For perfect junctions (i.e. hard-wall potential), this was considered by [ChLeBl] in the framework of scattering matrices. Here we allow for imperfections in the junction, so that the complex order parameter continues as a smooth function, which is a constant in the superconducting banks, and vanishes rapidly inside the lead. We obtain quantization rules for resonant Andreev states near energy $E$ close to the Fermi level, including the determination of the resonance width.
\end{abstract}

\section{Introduction.}

Bogoliubov-de Gennes Hamiltonian is a $2 \times 2$ matrix $\mathcal{P}(x, \xi)$ defined for $(x, \xi) \in T^{*} \mathbf{R}$, which describes the dynamics of a pair of quasi-particles (hole/electron) in a 1-D metallic lead connecting 2 superconducting contacts. Diagonal terms are of the form $\pm\left(\xi^{2}-\mu(x)\right)$, where $\mu(x)$ stands for the chemical potential, while the off-diagonal interaction with the supraconducting bulk is modeled through a complex potential, or superconducting gap, $\Delta_{0} e^{i \phi_{ \pm} / 2}$ at the boundary ; due to the finite range of the junction, we may consider that the interaction continues to a smooth function $x \mapsto$ $\Delta(x) e^{i \phi(x) / 2}$ on a neighborhood of the lead (say, the interval $[-L, L]$.) So we assume that $\Delta(x)$, we will call henceforth the "gap function", is a smooth positive function, increasing on $x>0$, with $\Delta(x)=0,|x| \leq x_{1}<L$ and $\Delta(x)=\Delta_{0},|x| \geq x_{2}>L$ (ignoring the fact that $\Delta$ shows typically isolated zeroes (vortices) in the supraconducting bank). In the same way, we will assume that $\phi(x)=\operatorname{sgn}(x) \phi$ takes only 2 values. The chemical potential $\mu(x)$ will be extended also to a smooth positive function on a neighborhood of $[-L, L]$, constant and $>\Delta_{0}$ for $|x| \geq x_{2}$. As is usual for a metal, we assume that $\mu$ and $\Delta$ are even in $x$, which provides this model with the CPT symmetry.

We introduce a "Planck constant" $h>0$, which stands for the ratio of $L$ to the characteristic de Broglie wave-length, and take usual $h$-Weyl quantization,

$$
\mathcal{P}\left(x, h D_{x}\right)=\left(\begin{array}{cc}
\left(h D_{x}\right)^{2}-\mu(x) & \Delta(x) e^{i \phi(x) / 2} \\
\Delta(x) e^{-i \phi(x) / 2} & -\left(h D_{x}\right)^{2}+\mu(x)
\end{array}\right)
$$

An electron $e^{-}$moving in the metallic lead with energy $0<E \leq \Delta$ (measured with respect to Fermi level $\left.E_{F}\right)$ and kinetic energy $K_{+}(x)=\mu(x)+\sqrt{E^{2}-\Delta(x)^{2}}$ is reflected back from the supraconductor as a hole $e^{+}$, with kinetic energy $K_{-}(x)=\mu(x)-\sqrt{E^{2}-\Delta(x)^{2}}$, injecting a Cooper pair into the bulk. When $\inf _{[-L, L]} \mu(x) \geq E$, and $\phi \neq 0$, this process yields so called phase-sensitive Andreev states, carrying supercurrents proportional to the $\phi$-derivative of the eigen-energies $E_{k}(h)$ of $\mathcal{P}\left(x, h D_{x}\right)$. Since 
$\mathcal{P}\left(x, h D_{x}\right)$ is self-adjoint, there is of course also an electron moving to the left, and a hole moving to the right (in fact, $\mathcal{P}\left(x, h D_{x}\right)$ is the Hamiltonian for 2 pairs of quasi-particles), for no net transfer of charge can occur through the lead in absence of thermalisation. So we stress that Bogoliubovde Gennes Hamiltonian is only a simplified model for superconductivity, and that a more thorough treatment should also take into account the self-consistency relations coupling the quasi-particle with the gap function $\Delta(x)$ and the phase $\phi(x)$, that we treat here as "effective potentials" (see [KeSo]).

In the case where $\Delta(x)$ is a "hard-wall" potential, this was studied in [ChLesBl], [CaMo] in the framework of scattering matrices. In [BeIfaRo], we derived semi-classical quantization rules for Andreev states near energy $E$, from a microlocal study of the Hamiltonian in the "inner region" $\Delta(x) \leq E$ alone. For simplicity, we assumed that $\Delta(x)$ varies linearly near $E$, namely if $\left.x_{0} \in\right] x_{1}, x_{2}$ [ is such that $\Delta\left(x_{0}\right)=E$, then $\mu(x)=\mu=$ Const. and $\Delta(x)=E+\alpha\left(x-x_{0}\right)$ near $x_{0}$.

Here we want to take also into account the "outer region" $\Delta(x) \geq E$ (i.e. $|x| \geq x_{0}$ ) of the junction, entering the supraconducting bulk. As a matter of fact, the microlocal solutions, purely oscillating in $\Delta(x) \leq E$, acquire a complex phase in $\Delta(x) \geq E$, which is of course related to phasespace tunneling. We make the assumption that the junction is extended, in such a way that the quasi-particle turns into a resonant state before creating a new Cooper pair, its dynamics still being governed by Bogoliubov-de Gennes Hamiltonian. So we assume that $\mu(x)$ and $\Delta(x)$ are defined on the entire real line, taking constant values for $|x| \geq x_{2}>L$, so that (0.1) can be defined as a self-adjoint operator on $L^{2}(\mathbf{R}) \otimes \mathbf{C}^{2}$. We will translate the usual theory of analytic dilations [ReSi] in the context of CPT symmetry, and find semi-classical resonances near a "scattering" Andreev level, i.e. complex correction to the real eigen-energies $E_{k}(h)$ of $\mathcal{P}\left(x, h D_{x}\right)$.

\section{1) The real part of the resonances.}

The bicharacteristic set in $\{\xi>0\}$ at energy $E$, of the form $\operatorname{det} \mathcal{P}(x, \xi)-E=0$, or $\xi^{2}=K_{ \pm}(x)$, consists of : (1) two real curves $\rho_{ \pm}$over $\left[-x_{0}, x_{0}\right]$, joining smoothly to a close curve at the "branching points" $a^{\prime}=\left(-x_{0}, \xi_{0}\right)$ and $a=\left(x_{0}, \xi_{0}\right)$ (so to make $\rho_{+} \cup \rho_{-}$diffeomorphic to $\mathbf{S}^{1}$ ); (2) complex branches $\rho_{ \pm}^{<}$over $\left.]-\infty,-x_{0}\right]$, and $\rho_{ \pm}^{>}$over $\left[x_{0},+\infty\right.$ [ respectively. They all have a vertical tangent at $a, a^{\prime}$. We complete this picture by reflection on the $x$ axis, denoting the corresponding branching points by $b^{\prime}, b$.

\section{a) Microlocal solutions supported on $\rho_{ \pm}$}

First we recall from [3] the construction of distributions microlocalized on the Lagrangians $\rho_{ \pm}$, and verifying the PT symmetries of the problem. We denote the parity operator by $\vee: u(x) \rightarrow u(-x)$, and the time reversal operator by $\mathcal{I}: u(x) \rightarrow \overline{u(x)}$.

Definition 1.1: We call "admissible $\mathbf{C}^{2}$-valued Lagrangian distribution" an oscillatory integral

$$
I(S, \varphi)(x, h)=(2 \pi h)^{-d / 2} \int_{\mathbf{R}^{d}} e^{i \varphi(x, \Theta, h) / h} S(x, \Theta ; h) d \Theta
$$

with the following properties : (1) $\varphi(x, \Theta, h)$ denotes a non degenerate phase-function, and

$$
S(x, \Theta ; h)=S_{0}(x, \Theta ; h)+h S_{1}(x, \Theta ; h)+\cdots
$$


a $\mathbf{C}^{2}$-valued amplitude (i.e. a classical symbol in $h$ ), $S_{0}=\left(\begin{array}{c}e^{i \phi / 2} X \\ Y\end{array}\right)$ possibly depending on $h$ (with the property that $\phi(x)=\operatorname{sgn}(x) \phi)$; (2) The symbols $X=X(x, \Theta, h), Y=Y(x, \Theta, h)$ have their principal part $\left(\begin{array}{c}X_{0} \\ Y_{0}\end{array}\right)=\lambda(x, \Theta ; h)\left(\begin{array}{c}X_{0}^{\prime} \\ Y_{0}^{\prime}\end{array}\right), \lambda \in \mathbf{C}$, proportional to a real vector $\left(\begin{array}{c}X_{0}^{\prime} \\ Y_{0}^{\prime}\end{array}\right)$, depending also on $(x, \Theta ; h)$.

Of course, all these functions may depend on additional parameters. One of the main problem consists in finding microlocal solutions near the branching points $a, a^{\prime}$. Due to PT symmetry, it suffices to focus on $a=\left(x_{0}, \xi_{0}\right)$. In $h$-Fourier representation, the Hamiltonian takes the form

$$
\mathcal{P}^{a}\left(-h D_{\xi}, \xi\right)=\left(\begin{array}{cc}
\xi^{2}-\mu & e^{i \phi / 2}\left(E-\alpha h D_{\xi}-\alpha x_{0}\right) \\
e^{-i \phi / 2}\left(E-\alpha h D_{\xi}-\alpha x_{0}\right) & -\xi^{2}+\mu
\end{array}\right)
$$

where $\mu=\xi_{0}^{2}$ is a constant, equal to the value of the chemical potential at $x_{0}$. Consider the equation $\left(\mathcal{P}^{a}\left(-h D_{\xi}, \xi\right)-E\right) \widehat{U}=0$, where $\widehat{U}=\left(\begin{array}{c}\widehat{\varphi}_{1} \\ \widehat{\varphi}_{2}\end{array}\right)$. Clearly, the system decouples, and to account for timereversal symmetry, it is convenient to introduce the scaling parameter $\beta=\sqrt{\alpha}\left(2 \xi_{0}\right)^{-3 / 2}$, together with the changes of variables $\xi=\xi_{0}\left( \pm 2 \beta \xi^{\prime}+1\right)$. The functions $\widetilde{u}_{ \pm \beta}\left(\xi^{\prime}\right)=\left(\xi^{2}-\mu-E\right)^{-1 / 2} e^{-i\left(E-\alpha x_{0}\right) \xi / \alpha h} \widehat{\varphi}_{2}$ satisfy a second order ODE of the form

$$
\left(\widetilde{P}_{ \pm \beta}\left(-h D_{\xi^{\prime}}, \xi^{\prime}, h\right)-\frac{E_{1}^{2}}{\beta^{2}}\right) \widetilde{u}_{ \pm \beta}\left(\xi^{\prime}\right)=0
$$

with $E_{1}=\left(2 \xi_{0}\right)^{-2} E$, and

$$
\begin{aligned}
\widetilde{P}_{ \pm \beta}( & \left.-h D_{\xi^{\prime}}, \xi^{\prime}, h\right)=\left(h D_{\xi^{\prime}}\right)^{2}+\left(\xi^{\prime} \pm \beta \xi^{\prime 2}\right)^{2} \\
& +h^{2}\left(2 \xi_{0}\right)^{-2} \beta^{2}\left(2 \beta^{2} \xi^{\prime 2} \pm 2 \beta \xi^{\prime}+\frac{3}{4}+E_{1}\right)\left(\beta^{2} \xi^{\prime 2}+\beta \xi^{\prime}-E_{1}\right)^{-2}
\end{aligned}
$$

Operators $\widetilde{P}_{\beta}$ and $\widetilde{P}_{-\beta}$ are unitarily equivalent, and so have the same spectrum. Up to the $\mathcal{O}\left(h^{2}\right)$ term, $\widetilde{P}_{ \pm \beta}\left(-h D_{\xi^{\prime}}, \xi^{\prime}, h\right)$ have the structure of an "anharmonic oscillator", with "potential wells" at $\xi^{\prime}=0, \mp 1 / \beta$ separated by a "barrier" at $\xi^{\prime}=\mp 1 /(2 \beta)$. It is also well known [HeSj] that, viewed as a $h$-PDO of order 0 , microlocally defined near $\left(x^{\prime}, \xi^{\prime}\right)=0, \widetilde{P}=\widetilde{P}_{ \pm \beta}$ can be taken to the normal form of a harmonic oscillator, away from the "barrier". More precisely, there exists a real-valued analytic symbol $F(t, h)=F_{ \pm \beta}(t, h) \sim \sum_{j=0}^{\infty} F_{j}(t) h^{j}$, defined for $t \in \operatorname{neigh}(0), F_{0}(0)=0, F_{0}^{\prime}(0)=\frac{1}{2}$, $F_{1}(t)=$ Const., and (formally) unitary FIO's $A=A_{ \pm \beta}$ whose canonical transformations $\kappa_{A}$ defined in a neighborhood of $(0,0)$, are close to identity and map this point onto itself, such that

$$
A^{*} F(\widetilde{P}, h) A=P_{0}=\frac{1}{2}\left(\left(h D_{\eta}\right)^{2}+\eta^{2}-h\right)
$$

Define the large parameter $\nu$ by $F\left(\frac{E^{2}}{2 \xi_{0} \alpha}, h\right)=\nu h$. So $\widetilde{u}=\widetilde{u}_{ \pm}$solves $(1.2)$ microlocally near $(0,0)$ iff $v=A^{*} \widetilde{u}$ solves Weber equation $\left(P_{0}-\nu h\right) v=0$ microlocally near $(0,0)$, when $\nu h \sim \frac{E^{2}}{4 \xi_{0} \alpha}$ is small enough. The well known parabolic cylinder functions $D_{\nu}$ and $D_{-\nu-1}$, provide with a basis of solutions of $\frac{1}{2}\left(\left(h D_{\eta}\right)^{2}+\eta^{2}-h\right) v=\nu v$. We shall use $D_{-\nu-1}$, and write

$$
v=A^{*} \widetilde{u}_{ \pm \beta}=\sum_{\varepsilon= \pm 1} \alpha_{\varepsilon, \pm \beta}^{(-\nu-1)} D_{-\nu-1}\left(i \varepsilon(h / 2)^{-1 / 2} \eta\right)
$$


for complex constants $\alpha_{\varepsilon, \pm \beta}^{(-\nu-1)}$.

These microlocal solutions can be expressed in the spatial representation by taking inverse $h$ Fourier transformation ; expanding integrals of the type (1.4) by stationary phase, both pieces of bicharacteristics $\rho_{ \pm}$contribute to $U_{\varepsilon, \pm \beta}$ near $a$. Microlocal solutions near $a^{\prime}$ are deduced by PT symmetry.

Once microlocal solutions $U_{\varepsilon, \beta}^{a,-\nu-1}$ have been obtained that way near the branching point $a$, it is standard to extend them up to $a^{\prime}$ as WKB solutions $\left(U_{\varepsilon, \beta}^{a,-\nu-1}\right)_{\text {ext }}$, taking advantage that $\mathcal{P}$ has simple characteristics away from $a, a^{\prime}$. When $\Delta(x) \equiv 0$, i.e. for $-x_{1} \leq x \leq x_{1}$, they are completely decoupled, which means that the solution is either a pure electronic state, i.e. colinear to the vector $\left(\begin{array}{l}1 \\ 0\end{array}\right)$ of $\mathbf{C}^{2}$, or pure hole state, i.e. colinear to $\left(\begin{array}{l}0 \\ 1\end{array}\right)$. Otherwise, they are a superposition of electronic/hole states. We summarize these constructions in the :

Proposition 1.2: For $x<x_{0}$ near $x_{0}$, there are 2 basis of oscillating microlocal solutions of $\left(\mathcal{P}^{a}-\right.$ $E) U=0$ indexed by $\varepsilon= \pm 1$ :

$$
\sum_{\rho= \pm 1} U_{\rho, \varepsilon, \pm \beta}^{a, \nu}\left(x, h^{\prime}\right), \quad \sum_{\rho= \pm 1} U_{\rho, \varepsilon, \pm \beta}^{a,-\nu-1}\left(x, h^{\prime}\right)
$$

Here the branch with $\rho=\rho_{ \pm}= \pm 1$ is microlocalized on $\rho_{ \pm}$, i.e. the part on $\rho_{+}\left(\xi>\xi_{0}\right.$ near a), belongs to the electron state, while the part $\rho_{-}\left(\xi<\xi_{0}\right.$ near a) belongs to the hole state; they satisfy, for $\rho= \pm$ :

$$
U_{\rho,-, \beta}^{a,-\nu-1}=U_{\rho,+, \beta}^{a,-\nu-1}+\mathcal{O}(h)
$$

and

$$
U_{-, \varepsilon, \beta}^{a,-\nu-1}=U_{+, \varepsilon,-\beta}^{a,-\nu-1}
$$

Each of these solutions is an admissible $\mathbf{C}^{2}$-valued lagrangian distribution in the sense of Definition 1.1. Divide all microlocal solutions by the trivial factor $e^{i \pi / 4} e^{i E_{0} \xi_{0} / h^{\prime}}, E_{0}=E-\alpha x_{0}$. Then with the notations of (1.4) the general solution of $\left(\mathcal{P}^{a}-E\right) U=0$ is of the form

$$
U=\sum_{\rho, \varepsilon} \alpha_{\varepsilon, \pm \beta}^{(-\nu-1)} U_{\rho, \varepsilon, \pm \beta}^{a,-\nu-1}
$$

The solutions near $a^{\prime}$ are given by symmetry, e.g. $U_{\rho, \varepsilon,-\beta}^{a^{\prime},-\nu-1}={ }^{\vee} \mathcal{I} U_{\rho, \varepsilon, \beta}^{a,-\nu-1)}$. Moreover both microlocal families can be extended as WKB solutions (satisfying Definition 1.1) along the bicharacteristics.

Note also that in this region where $\mu(x)$ is a constant, $U_{\rho, \varepsilon, \pm \beta}=e^{i x \xi_{0} / h} \mathcal{U}_{\rho, \varepsilon, \pm \beta ; h^{\prime}}$ with $\mathcal{U}_{\rho, \varepsilon, \pm \beta ; h^{\prime}}$ oscillating on a frequency scale $1 / h^{\prime}=1 /(\alpha h)$, so if we think of the slope $\alpha$ to be large, $U_{\rho, \varepsilon, \pm \beta}$ behaves as a plane wave $e^{i x \xi_{0} / h}$, modulated by a slow varying function.

\section{b) Real holonomy and approximate Bohr-Sommerfeld quantization condition.}

The microlocal kernel $K_{h}(E)$ of $\mathcal{P}-E$ on $]-x_{0}, x_{0}\left[\times \mathbf{R}_{+}\right.$can be viewed as a 4 -D fibre vector bundle $\mathcal{F}_{h}(E)$ of admissible Lagrangian distributions over $\mathbf{S}^{1}$. We characterize the real part of the 
resonances as the set of $E_{k}(h)$ near $E$ such that this fibre bundle is trivial. We start by computing the holonomy of that bundle.

First we normalize the basis in $K_{h}(E)$ obtained in Proposition 1.2 using generalized Wronskians introduced in $[\mathrm{HeSj}]$, [Ro]. Namely, let $\chi=\chi^{a}$ be a smooth cut-off supported on a sufficiently small neighborhood of $a$, equal to 1 near $a, \omega_{ \pm}=\omega_{ \pm}^{a}$ a small neighborhood of $\rho_{ \pm} \cap \operatorname{supp}\left[\mathcal{P}, \chi^{a}\right]$, and $\chi_{\omega_{ \pm}}=\chi_{\omega_{ \pm}}^{a}$ a cut-off equal to 1 near $\omega_{ \pm}$. We take Weyl $h$-quantization of these symbols, and for $U, V \in K_{h}(E)$, we call

$$
\mathcal{W}_{\omega_{ \pm}}(U, V)=\left(\chi_{\omega_{ \pm}} \frac{i}{h}[\mathcal{P}, \chi] U \mid V\right)=\left(\chi_{\omega_{ \pm}} \frac{i}{h}[\mathcal{P}, \chi] \widehat{U} \mid \widehat{V}\right)
$$

the microlocal Wronskian of $(U, \bar{V})$ in $\omega_{ \pm}$. This is a sesquilinear form on $K_{h}(E)$, and $\mathcal{W}_{\omega_{ \pm}}(U, U)$ is independent, modulo error terms $\mathcal{O}\left(h^{\infty}\right)$, of the choices of $\chi^{a}$ and $\chi_{\omega_{ \pm}}^{a}$ as above. Taking into account both contributions of $\rho_{ \pm}$we define also

$$
\mathcal{W}(U, V)=\mathcal{W}_{\omega_{+}}(U, V)+\mathcal{W}_{\omega_{-}}(U, V)
$$

For each microlocal solution $\widehat{U}=\widehat{U}_{\varepsilon, \pm \beta}^{a,-\nu-1}$, it turns out that $\mathcal{W}(U, U)$ have asymptotic expansions in $h^{\prime}$, of the form $w_{0}(E, \beta)+h^{\prime} w_{1}(E, \beta)+\cdots$, with $w_{0}(E, \beta)>0$.

Given $\chi=\chi^{a}$, let now $\tilde{\chi}=\widetilde{\chi}^{a}$ be a new cut-off equal to 1 on the support of $\chi^{a}$, and to 0 outside a slightly larger set. For $U, V \in K_{h}(E)$ we set $(U \mid V)_{\tilde{\chi}}=(\widetilde{\chi} U \mid V)$. Then it is easy to see that there is an orthonormal basis of $K_{h}(E)$ for the "scalar product" $(U \mid V)_{\tilde{\chi}}$, which is at the same time orthogonal for $\mathcal{W}(U, V)$ (everything being defined modulo $\mathcal{O}\left(h^{\infty}\right)$.) This allows to find $V_{\varepsilon}=V_{\varepsilon, \beta}^{a,-\nu-1}$ of the form (1.7) such that $\left(V_{\varepsilon} \mid V_{\varepsilon^{\prime}}\right)_{\tilde{\chi}}=\delta_{\varepsilon, \varepsilon^{\prime}},\left(\varepsilon, \varepsilon^{\prime}= \pm 1\right), \mathcal{W}\left(V_{ \pm}, V_{ \pm}\right)>0$, and $\mathcal{W}\left(V_{+}, V_{-}\right)=0$. Of course, by the symmetry ${ }^{\vee} \mathcal{I} \mathcal{P}\left(x, h D_{x}\right)=\mathcal{P}\left(x, h D_{x}\right) \mathcal{I}^{\vee}$, such normalized microlocal solutions exist as well near $a^{\prime}$. The Lagrangian distributions

$$
F_{\varepsilon, \beta}^{a,-\nu-1}=\chi_{\omega^{a}} \frac{i}{h}\left[\mathcal{P}, \chi^{a}\right] U_{\varepsilon, \beta}^{a,-\nu-1}
$$

and similarly $F_{\varepsilon,-\beta}^{a^{\prime},-\nu-1}$ span the microlocal co-kernel $K_{h}^{*}(E)$ of $\mathcal{P}-E$ in $]-x_{0}, x_{0}\left[\times \mathbf{R}_{+}\right.$, as $\varepsilon= \pm 1$. The same holds for or $G_{\varepsilon, \beta}^{a,-\nu-1}$ obtained by replacing $U_{\epsilon, \beta}^{a,-\nu-1}$ by the "orthonormal basis" $V_{\varepsilon, \beta}^{a,-\nu-1}$ as above.

Because of Proposition 1.2, the normalized microlocal solutions $V_{\varepsilon,-\beta}^{a^{\prime},-\nu-1}$ are related to the extension of the normalized microlocal solutions $V_{\varepsilon, \beta}^{a,-\nu-1}$ along the bicharacteristics by a monodromy matrix $M^{a, a^{\prime}}=\left(\begin{array}{ll}d_{11} & d_{12} \\ d_{21} & d_{22}\end{array}\right) \in U(2)$. Similarly, we obtain $M^{a^{\prime}, a}$ by extending from the left to the right, and due to symmetry, $M^{a^{\prime}, a}=\left(M^{a, a^{\prime}}\right)^{-1}=\left(M^{a, a^{\prime}}\right)^{*}$. Diagonal entries of these matrices are given by action integrals along $\rho_{ \pm}$(see e.g. [Ro]). Off-diagonal terms are $\mathcal{O}\left(h^{\prime}\right)$ and can be computed with the help of the Wronskian (in the ordinary sense) associated with the system $(\mathcal{P}-E) U=0$ (see e.g. $[\mathrm{Ba}])$.

The quantization condition is satisfied, precisely when the rank of that system drops of one unit (actually, because of degeneracy, of 2 units), i.e. when $\operatorname{dim} K_{h}(E)=\operatorname{dim} K_{h}^{*}(E)=2$. This amounts to set to zero the determinant of some $\operatorname{Gram}$ matrix $\operatorname{Gram}(E, h)$ expressed in the basis 
$\left(V_{\varepsilon, \beta}^{a,-\nu-1}, V_{\varepsilon^{\prime},-\beta}^{a^{\prime},-\nu-1}\right)$ and $\left(G_{\varepsilon, \beta}^{a,-\nu-1}, G_{\varepsilon^{\prime},-\beta}^{a^{\prime},-\nu-1}\right)$. So $E=E_{k}(h)$ is an eigenvalue, modulo $\mathcal{O}\left(h^{\infty}\right)$, of $\mathcal{P}\left(x, h D_{x}\right)$, corresponding to an Andreev state, iff $\operatorname{det} \operatorname{Gram}(E, h)=0$.

Here we note the sensitivity of the energy levels $E_{k}(h)$ with respect to $\phi$. In the "hard-wall" limit $\alpha \rightarrow \infty$, we recover the quasi-particle spectrum, of the form $\cos \phi=\cos \left(\frac{g\left(E_{k}(h)\right)}{h}-2 \arccos \left(\frac{E_{k}(h)}{\Delta_{0}}\right)\right)$ for some smooth function $g$ (see [CayMon], [ChLesBl]).

\section{2) The imaginary part of the resonances.}

The considerations above are not sufficient to account for exponentially small corrections to $E_{k}(h)$. Further information will be extracted from a Grusin problem.

\section{a) Microlocal solutions with complex phase.}

Microlocal solutions, computed in the real phase space, are purely oscillating in the metallic lead $\left[-x_{0}, x_{0}\right]$. To get information in the "superconducting part of the junction", we need use "infinitesimal" invariance by time reversal and conjugation of charge. The substitutions $\xi^{\prime} \mapsto \pm i \xi^{\prime}$, or equivalently, $\beta \mapsto \pm i \beta$, leave invariant equation (1.1), with a new operator $\widetilde{P}_{ \pm i \beta}$ in Fourier-Laplace representation. Microlocal solutions of $\left(\widetilde{P}_{ \pm i \beta}-E\right) \widetilde{u}=0$ are constructed similarly and, on the real domain, independently of those of $\left(\widetilde{P}_{ \pm \beta}-E\right) \widetilde{u}=0$.

Thus the fibre bundle of microlocal solutions on $\mathbf{R} \times \mathbf{R}_{+}$(i.e. microlocal kernel of $\mathcal{P}-E$ ) splits as $\mathcal{F}_{h}^{<}(E) \oplus \mathcal{F}_{h}(E) \oplus \mathcal{F}_{h}^{>}(E)$, where we recall $\mathcal{F}_{h}(E)$ from Sect.1, and $\mathcal{F}_{h}^{<,>}(E)$ are 2-D (trivial) fibre bundles over $\mathbf{R}$.

Nevertheless, taking advantage that the coefficients are analytic near $a, a^{\prime}$, there is a way to couple $\mathcal{F}_{h}^{<,>}(E)$ with $\mathcal{F}_{h}(E)$ in the complex domain. This, together with the assignment that the global section be "outgoing" at infinity, accounts for complex holonomy.

First we investigate complex holonomy near $a$, and consider the family of operators, obtained by extending $\widetilde{P}_{ \pm \beta}$ along a path $\left\{e^{i \gamma} \beta, 0 \leq \gamma \leq 2 \pi\right\}$ in the complex plane ; similarly, we consider the family of Lagrangian distributions obtained by extending $\widetilde{u}_{\beta}\left(\xi^{\prime}\right)$ along that path. They will solve (1.1) iff $\gamma=0, \pm \pi / 2, \pi$.

These solutions are related through their Lagrangian manifolds as follows : consider (for simplicity) the principal part of $\widetilde{P}_{ \pm \beta}$ and $\widetilde{P}_{ \pm i \beta}$, namely $\widetilde{Q}_{\beta}\left(-h D_{\xi^{\prime}}, \xi^{\prime}\right)=\left(h D_{\xi^{\prime}}\right)^{2}+\left(\xi^{\prime}+\beta \xi^{\prime 2}\right)^{2}$ and $\widetilde{Q}_{i \beta}\left(-h D_{\xi^{\prime}}, \xi^{\prime}\right)=\left(h D_{\xi^{\prime}}\right)^{2}+\left(\xi^{\prime}+i \beta \xi^{\prime 2}\right)^{2}$. The potentials being equal for $\xi^{\prime}=0$ and $\xi^{\prime}=-2 /(1+i) \beta$, the real Lagrangian manifold $\rho_{+}$near $a$ extends analytically along the loop $\left\{e^{i \gamma} \beta: \gamma \in[0,2 \pi]\right\}$ in the complex domain, so that it intersects $\rho_{+}^{>}$at $-2 /(1+i) \beta$ for $\gamma=\pi / 2$. We can argue similarly for the other branches. Actually, both $\rho_{ \pm}$and $\rho_{ \pm}^{>}$are branches of a single 2-sheeted Riemann surface, with complex "turning points".

We can assign to this analytic manifold microlocal solutions for $\widetilde{P}_{e^{i \gamma} \beta}$ as in (1.2) with complex phase, which yields in turn solutions of $(\mathcal{P}-E) U=0$ for relevant values $0, \pm \frac{\pi}{2}, \pi$ of the parameter $\gamma$; these solutions are very similar to the $U_{\varepsilon, \pm \beta}^{a}$ 's given in Proposition 1.2. The monodromy operator, acting on microlocal solutions, is known as connection isomorphism, see [DeDiPh] and references therein, and also [Fe], or [Ro,Sect.4,g] in the case of a system. This connection isomorphism is given by a matrix $N^{a} \in U(2)$, whose entries are expressed in term of exponentials of action integrals computed along Stokes lines between the complex turning points. 
Let us consider next the conditions at infinity : for $|x|>x_{2}, \mathcal{P}$ has constant coefficients, so we make an analytic dilation of the form $x \mapsto \exp [(\operatorname{sgn} x) \vartheta] x, \vartheta>0$. Plane waves with positive momentum have the phase $\exp \left[i x\left(\xi_{1} \pm i \xi_{2}\right) / h\right]$ where $\xi_{1} \pm i \xi_{2}=\left(\mu_{0} \pm i \sqrt{\Delta_{0}^{2}-E^{2}}\right)^{1 / 2}$, according to the choice of $\rho_{ \pm}^{<,>}$. Analytic distorsion is turned on for $|x|$ large enough, and $\vartheta$ in the complex upperhalf plane. We denote by $\mathcal{P}_{\vartheta}$ the distorted operator. So for all $\operatorname{Im} \vartheta \geq 0$ small enough, we can make the "electronic state" (resp. "hole state") exponentially decaying at $+\infty$ (resp. $-\infty$ ), which models the scattering process $e^{+} \rightarrow e^{-}$, and similarly for the scattering process $e^{-} \rightarrow e^{+}$, thus preserving conservation of charge.

\section{b) A Grusin problem and the width of resonances.}

Following a classical procedure in Fredholm theory, we can translate the original eigenvalue problem for $\mathcal{P}$ into a finite dimensional problem via the Grusin operator [HeSj3,Sect4] ; this is essentially the isomorphism $\left(H^{2}(\mathbf{R}) \otimes \mathbf{C}^{2}\right) / \widetilde{K}_{h}(E) \rightarrow \operatorname{Ran}(\mathcal{P}-E) \subset L^{2}(\mathbf{R}) \otimes \mathbf{C}^{2}$. Here $\widetilde{K}_{h}(E)$ denotes the 6-D microlocal kernel of $\mathcal{P}-E$ in $\mathbf{R} \times \mathbf{R}_{+}$, restricted to the set of outgoing functions defined above. For $\mathcal{P}=\mathcal{P}_{\vartheta}$, we consider $\mathcal{G}(E)=\mathcal{G}(\vartheta, E)$ of the form :

$$
\begin{gathered}
\mathcal{G}(E)=\left(\begin{array}{cc}
\mathcal{P}-E & R_{-} \\
R_{+} & 0
\end{array}\right):\left(H^{2}(\mathbf{R}) \otimes \mathbf{C}^{2}\right) \times \mathbf{C}^{6} \rightarrow\left(L^{2}(\mathbf{R}) \otimes \mathbf{C}^{2}\right) \times \mathbf{C}^{6} \\
R_{-}\left(x_{1}, \cdots, x_{6}\right)=\sum_{j=1}^{6} x_{j} G_{j}, \quad R_{+} U=\left(\left(U \mid G_{j}\right)\right)_{1 \leq j \leq 6}
\end{gathered}
$$

where the $G_{j}$ 's range over the basis of co-kernel $\widetilde{K}_{h}^{*}(E)$ consisting of $G_{\varepsilon, \beta}^{a}, G_{\varepsilon,-\beta}^{a^{\prime}}, G_{+, i \beta}^{a}, G_{-,-i \beta}^{a^{\prime}}$ (or their analytic continuation at the branching points).

At this point we make the following remark : Since our Grusin operator (2.1) involves only positive frequencies, it cannot be associated with the self-adjoint operator $\mathcal{P}_{\vartheta}$ (for real $\vartheta$ ). But resonances are due precisely to a breaking of time-reversal symmetry, and their imaginary part is computed by introducing a $h$-Pseudo-differential cutoff $\Phi\left(x, h D_{x}\right)$ supported in $\{\xi>0\}$. Because negative frequencies will be eventually removed, we may best think of (2.1) as a short-hand notation for the "full" Grusin operator $\mathcal{G}(E)$, that would take into account the negative frequencies as well.

For all $h>0$ small enough, $\mathcal{G}(E)$ is bijective, with bounded inverse

$$
\mathcal{E}(E)=\left(\begin{array}{cc}
\mathcal{E}_{0}(E) & \mathcal{E}_{+}(E) \\
\mathcal{E}_{-}(E) & \mathcal{E}_{-+}(E)
\end{array}\right)
$$

and has the property, that $E$ is an eigenvalue of $\mathcal{P}$ iff $\operatorname{det} \mathcal{E}_{-+}(E)=0$. The construction of $\mathcal{E}(E)$ is carried as in $[\mathrm{HeSj}],[\mathrm{Ro}]$, selecting solutions according to the prescriptions above. Matrix $\mathcal{E}_{-+}(E)$ decouples modulo $\mathcal{O}\left(h^{\infty}\right)$, with a $4 \times 4$ block conjugated to $\operatorname{Gram}(E)$; the interaction with the "incoming hole" and "outgoing electron" occurs through the "turning points" in the complex domain, involving the connection isomorphisms $N^{a}, N^{a^{\prime}}$.

For complex $(\vartheta, E)$, we note that $\left(\mathcal{P}_{\vartheta}-E\right)^{*}=\mathcal{P}_{\bar{\vartheta}}-\bar{E}$. Applying distorsion to the Grusin operator as well, we get :

$$
\mathcal{G}(\vartheta, E)=\left(\begin{array}{cc}
\mathcal{P}_{\vartheta}-E & R_{-}(\vartheta, E) \\
R_{+}(\vartheta, E) & 0
\end{array}\right), \quad \mathcal{E}(\vartheta, E)=\left(\begin{array}{cc}
\mathcal{E}_{0}(\vartheta, E) & \mathcal{E}_{+}(\vartheta, E) \\
\mathcal{E}_{-}(\vartheta, E) & \mathcal{E}_{-+}(\vartheta, E)
\end{array}\right)
$$


We can prove that $\mathcal{G}(\vartheta, E)$ is well-posed for all $\vartheta \in \mathbf{C}$ small enough, with inverse $\mathcal{E}(\vartheta, E)$. Recall from [Ro,Prop.7.1] the following identity :

Proposition 2.1: Let $\Phi \in C_{0}^{\infty}\left(\mathbf{R}^{2} ; \mathbf{R}\right)$. With the notations above

$$
\begin{aligned}
& {\left[R_{-}(\bar{\vartheta}, \bar{E})^{*} \Phi \mathcal{E}_{+}(\vartheta, E)\right]^{*} \mathcal{E}_{-+}(\bar{\vartheta}, \bar{E})-\left(\left[R_{-}(\vartheta, E)^{*} \Phi \mathcal{E}_{+}(\bar{\vartheta}, \bar{E})\right]^{*} \mathcal{E}_{-+}(\vartheta, E)\right)^{*}} \\
& \quad=\mathcal{E}_{+}(\vartheta, E)^{*}\left[\mathcal{P}_{\bar{\vartheta}}, \Phi\right] \mathcal{E}_{+}(\bar{\vartheta}, \bar{E})
\end{aligned}
$$

In the self-adjoint case, the corresponding statement would be " $\left(R_{-}^{*} \mathcal{E}_{+}\right)^{*} \mathcal{E}_{-+}$is self-adjoint". The determination of the width of resonances then goes as in [Ro], though it is somewhat more complicated due to the structure of $\mathcal{E}_{-+}(\vartheta, E)$. Take $W(\vartheta, E) \in \operatorname{Ker} \mathcal{E}_{-+}(\vartheta, E)$, and set $\mathcal{A}(\vartheta, E)=$ $\left[R_{-}(\vartheta, E)^{*} \Phi \mathcal{E}_{+}(\bar{\vartheta}, \bar{E})\right]^{*}$. From $(2.2)$ and the identity

$$
\left(W(\vartheta, E) \mid \mathcal{A}(\vartheta, E) \mathcal{E}_{-+}(\vartheta, E) W(\vartheta, E)\right)-\left(\mathcal{A}(\vartheta, E) \mathcal{E}_{-+}(\vartheta, E) W(\vartheta, E) \mid W(\vartheta, E)\right)=0
$$

we get

$$
\begin{aligned}
& \left(\mathcal{A}(\bar{\vartheta}, \bar{E}) \mathcal{E}_{-+}(\bar{\vartheta}, \bar{E}) W(\vartheta, E) \mid W(\vartheta, E)\right)-\left(\mathcal{A}(\vartheta, E) \mathcal{E}_{-+}(\vartheta, E) W(\vartheta, E) \mid W(\vartheta, E)\right) \\
& =\left(\mathcal{E}_{+}(\vartheta, E)^{*}\left[\mathcal{P}_{\bar{\vartheta}}, \Phi\right] \mathcal{E}_{+}(\bar{\vartheta}, \bar{E}) W(\vartheta, E) \mid W(\vartheta, E)\right)
\end{aligned}
$$

Evaluating both members of this equality gives an implicit equation for the imaginary part of the resonance, showing that behaves like $\exp \left[-2 \int_{\tau} \xi d x / h^{\prime}\right]$, where $\tau \subset \mathbf{C}$ is a path connecting the complex branching points in $\rho_{+}^{>} \cap \rho_{+}$.

\section{References:}

[An] A.Andreev. Zh. Eksp. Teor. Fiz., 46, p.1823 (1964) [Sov. Phys. JETP 19, p.1228 (1964)]

[Ba] H.Baklouti. Asymptotique des largeurs de résonances pour un modèle d'effet tunnel microlocal. Ann. Inst. H. Poincaré (Physique Théorique) 68(2), p.179-228 (1998)

[BeIfaRo] A.Bensouissi, A.Ifa, M.Rouleux. Andreev reflection and the semi-classical Bogoliubov-De Gennes Hamiltonian. Proceedings "Days of Diffraction 2009", Saint-Petersburg. p.37-42. Submitted. [CaMo] J.Cayssol, G.Montambaux. Exchange induced ordinary reflection in a single-channel SFS junction. Phys.Rev. B70, 224520 (2004).

[ChLeBl] N.Chtchelkatchev, G.Lesovik, G.Blatter. Phys.Rev.B, Vol.62, No.5, p.3559-3564 (2000)

[DePh] E.Delabaere, F.Pham. 1. Exact semiclassical expansions for 1-D quantum oscillators. J.Math. Phys. 38,(12), p.6128-6184 (1997) 2. Resurgence methods in semi-classical asymptotics. Ann. Inst. H.Poincaré 71(1), p.1-94, (1999).

[Fe] M.Fedoriouk. Méthodes Asymptotiques pour les Equations Différentielles Ordinaires. Editions MIR, Moscou (1987)

[HeSj] B.Helffer, J.Sjöstrand. 1. Analyse semi-classique pour l'equation de Harper. Mémoire (nouvelle série) No 3, Soc. Math. de France, 116 (4) (1986). 2. Analyse semi-classique pour l'equation de Harper II. Comportement semi-classique pres d'un rationnel. Mémoire (nouvelle série) No 40, Soc. Math. de France, 118 (1) (1989). 3. Semi-classical analysis for Harper's equation III. Mémoire No 39, Soc. Math. de France, 117 (4) (1988) 
[KeSo] J.B.Ketterson, S.N.Song. Superconductivity. Cambridge Univ. Press (1999)

[ReSi] M.Reed, B.Simon. Methods of Modern Math. Phys. Vol IV. Analysis of Operators. Academic Press (1975)

[Ro] M.Rouleux. Tunneling effects for $h$-Pseudodifferential Operators,... in: Evolution Equations, Feshbach Resonances, Singular Hodge Theory. Adv. Part. Diff. Eq. Wiley-VCH (1999)

[Sj] J.Sjöstrand. Singularités analytiques microlocales, Astérisque No.95 (1982). 